(C) 2009 IEEE. Personal use of this material is permitted. Permission from IEEE must be obtained for all other uses, in any current or future media, including reprinting/republishing this material for advertising or promotional purposes, creating new collective works, for resale or redistribution to servers or lists, or reuse of any copyrighted component of this work in other works. 


\title{
3D Virtual Worlds Enriching Innovation and Collaboration in Information Systems Research, Development, and Commercialisation
}

\author{
Carl Dreher ${ }^{1}$, Torsten Reiners ${ }^{1,2}$, Naomi Dreher ${ }^{1}$, and Heinz Dreher ${ }^{1}$, Member, IEEE \\ ${ }^{1}$ Curtin University of Technology, Western Australia, e-mails: c.dreher@curtin.edu.au, n.dreher@curtin.edu.au, \\ h.dreher@curtin.edu.au \\ ${ }^{2}$ Institute of Information Science, University of Hamburg, Hamburg, Germany, e-mail: reiners@econ.uni-hamburg.de
}

\begin{abstract}
The increasing rate of technological development is changing how we interact socially, commercially, and politically, making this an exciting yet challenging time in which to live. As the pace of development is destabilising the status quo, it is increasingly important for individuals and organisations alike to adapt. Research, development, and commercialisation are important tools in facilitating innovation for organisations engaging in the modern knowledge/networked economy. As an emergent player in the networked economy, Digital Ecosystems have great potential for innovation. In particular 3D Virtual Worlds offer exciting opportunities for Information Systems research, development, and commercialisation. By their very structure, 3D Virtual Worlds such as Second Life provide a powerful context for innovation and collaboration: Second Life users are immersed in a 3D environment and empowered by a menu-based object-builder and a programming language; in-built text, voice, and body-language communication tools enrich communication, collaboration, and help provide a rich contextual presence. The community of users apply themselves to all realms of human endeavour, from recreational socialisation to systems development and entrepreneurial ventures. The current paper describes a university research commercialisation systems development project (of an Automated Essay Grading system called MarkIT), that utilises a 3D Digital Ecosystem for both promotion and implementation. Subsequently, this paper discusses the benefits, limitations, and suggestions for future innovation in research, development, and commercialisation of Information Systems incorporating 3D Virtual Worlds.
\end{abstract}

Index Terms-3D Virtual Worlds; 3D Digital Ecosystems; Information systems research; research commercialisation and knowledge transfer.

\section{THE FACE OF TECHNOLOGICAL INNOVATION AND SOCIAL CHANGE}

The pace of technological development is increasing exponentially: the industrial revolution marked the advent of increasingly rapid technological and social change [1]; with the dawn of the modern information age, Moore's Law quantifies this exponential trend in the context of the information technology $[2,3]$. Concomitant with technological advancement is sociological change: the modern knowledge economy is a revolution in the way we interact socially and commercially [4]. Information Communications Technology (ICT) is at the confluence of technological advances and revolutionary social change. Revolution being a politicised term for what we currently couch as 'innovation': making things better.

As read-write networked communications technology (currently, Web 2.0) becomes more powerful and ubiquitous, the balance of power is shifting from major institutions (corporate entities and government institutions) to- wards the people - that is to say, the collective voice of citizens is empowered by the capacity to: publish usergenerated content (e.g., personal websites, blogs, wikis, and multimedia content such as on YouTube), and; to communicate globally with any entity that has a presence on the Internet (i.e., individuals, corporations, or governments). While the abovementioned facts are well-known, their implications for social change are just beginning to be explored.

As ICT continues to advance, so will social change, thereby revolutionising our social institutions that regulate political governance, the economy, and social interaction [5]. Political governance is being changed by ICT-use: because networks enhance peer-to-peer communication, this gives citizens the capacity to unite and self-regulate independent of their governments. While still early days, this trend is already beginning. Large social movements have been organised with a technology as simple as textmessaging (e.g., political rallies against oppression in China [6]); this is greatly enhanced by utilities such as Twitter using Rich Site Summary (RSS) formats. Furthermore Australian politicians have begun utilising social networking utilities such as Facebook, yet endorse Internet censorship [7]. The advent of intelligent semantic analysis tools may soon enable meaningful, uncensored communication between millions of people via the aggregation of semantically similar messages from multiple users in blogs (potentially called massively-multiple communication services, MMCSs). Such technology would greatly leverage the communication, self-awareness and autonomy of previously disenfranchised populaces. A united citizenry can destabilise the balance of power that governments currently hold.

ICT is also changing commerce by enabling people the world over to access information, goods and services that were previously unavailable to them (e.g., Google's suite of services, Amazon.com, and eBay), thus increasing the competitive pressure on commercial organisations. The movement away from intellectual property rights and towards open-source software (see GNU; [8]) is further destabilising the status quo. This is compounded by the ease with which digital property can be obtained illegally in the software and entertainment industries (i.e., programs, music, movies, and games). These trends are forcing companies in the software and entertainment industries to change their business models (e.g., towards strategies dependent on the initial free-provision of goods/services in order to establish a customer-base that then generates revenue from advertising and/or charging for premium goods/services).

ICT is also revolutionising how we interact socially. So- 
cial utilities such as Facebook, MySpace, and 3D Virtual Worlds (e.g., World of Warcraft, Halo, Croquet, Second Life) are an emerging phenomenon that are rapidly being adopted by large segments of society (commonly thought to be younger generations, dubbed digital natives [9]). Such social utilities provide networked digital public spaces that are replacing the physical publics spaces (e.g., parks, town squares) in which we have traditionally gathered [9].

In short, the increasing rate and extent of sociotechnological change is affecting our social, commercial and political processes. These systems must adapt / innovate in order to remain effective. Research, development, and commercialisation can play an important role in this adaptation / innovation.

\section{THE ROLE OF RESEARCH COMMERCIALISATION IN SOCIO-TECHNOLOGICAL INNOVATION}

The social, commercial, and political change outlined above is a reality that will likely continue to accelerate. It has the potential to change our social structures in ways we cannot fully predict (e.g., decentralising educational institutions towards distributed industry-integrated learning; [5]). Survival in a changing environment requires adaptation: for individuals, this currently manifests as the need for lifelong learning; for corporate entities (e.g., financial institutions, insurance brokers, ICT companies, and educational organisations alike) this requires constant innovation via research, development, and commercialisation (of information, goods or services). That is to say, as the pace of change increases in our modern economy, the role of research, innovation, and commercialisation will have increasing importance for the adaptation and survival of institutions (commercial institutions certainly, but also social and political institutions as outlined above). Indeed the Australian Government has recognised the importance of research commercialisation in the modern knowledge economy through its National Research Priorities [10].

A number of government-commissioned reports acknowledge the importance of research commercialisation [11-13]. Knowledge transfer has been defined as the process of research commercialisation whereby knowledge, intellectual property, or inventions are transformed into economic and social benefits for individuals, corporations, and society in general [12]. It is consequently an intrinsic aspect of the Australian Government's National Research Priorities [13]. Knowledge transfer can include a variety of processes: knowledge access (free distribution of knowledge through such means as publications and conferences); knowledge production (selling knowledge products via spin-out ventures, licensing, patents, or selling publications etc.); knowledge relationships (selling knowledge services through consulting or contractual relationships), and; knowledge engagement (longer term collaboration between academics and industry or community for mutual benefit) [13].

Knowledge transfer and research commercialisation may take place within the context of universities, governmentsponsored research centres, and commercial entities (including sole-traders, small-to-medium enterprises, and multinational companies). In many respects, the presence of ubiquitous powerful ICT in the networked economy can level the playing field such that a valued product can be leveraged with less capital expenditure than was previously required (e.g., Google [14]). This increased accessibility of ICT has lead to widespread creation of networked communities - what are now being called Digital Ecosystems.

\section{DigitAl ECOSYSTEMS: 2D AND 3D VIRTUAL ENVIRONMENTS}

Digital ecosystems are one of the prime ways in which ICT is changing how humans interact online [15-17]. They comprise both 2D interfaces (through Web 2.0 applications such as MySpace, Facebook, Flickr, and Twitter), as well as 3D interfaces (through Virtual Worlds such as World of Warcraft, Croque, and Second Life).

Indeed 3D Virtual Worlds offer rich potential which is still being explored and developed [18]. 3D Virtual Worlds have been described as "online environments that have game-like immersion and social media functionality without game-like goals or rules. At the heart is a sense of presence with others at the same time and in the same place” [19]. These qualities of immersion and presence are facilitated via an avatar, a user's in-world representation (usually a humanoid figure) that enables navigation and interaction in the 3D environment. The content of 3D Virtual Worlds ranges from developer-generated (e.g., Halo) to predominantly user-generated (e.g., Second Life). For instance, Second Life facilitates user-generated content via: a menubased 3D object creation tool, and; a programming language (the Linden Scripting Language, LSL). Similarly, the cost-of-use varies: some Virtual Worlds operate on a feefor-use basis (e.g., the BarbieGirls.com virtual world, or World of Warcraft), while others offer free entry (e.g., Second Life provide free avatar accounts and a freeware viewer client).

3D Virtual Worlds offer an unprecedented opportunity to engage in a Web-based digital environment that mimics the physical environment in which we have evolved. Humans have evolved as social beings in a 3D world governed by physical laws (e.g., time, space, gravity). We are predisposed to interact in this environment (i.e., to pay attention to faces, to perceive body-language, to desire social interaction [20]). Humans have enthusiastically adopted 2D digital ecosystems [9]; this trend is continuing with 3D digital ecosystems, which offer the capacity for more natural interaction by re-creating our physical world in a virtual environment. Specifically, 3D Virtual Worlds are unique because they: simulate both the visual appearance and functionality of the physical world, and; comprise a real community of users who (in some Virtual Worlds) engage in a virtual economy (using in-world currency that is exchangeable for real-world currency). For instance, Second Life has a vibrant in-world economy, real-estate market [21], and a simulated stock market called SL Capital Exchange. This creates a complex, self-regulating ecosystem comprising autonomous agents (i.e., humans represented by avatars), and means that Virtual Worlds are more than just simulation-technology, they literally are 3D Digital Ecosystems. Indeed, the Virtual World interfaces extend users' capacity to interact and increase their freedom of expression to ex- 
plore the gamut of human endeavour, including: artistic creation, recreation, social interaction, educational pursuits, collaborative developments (e.g., information systems), and entrepreneurial activities. Furthermore Virtual Worlds are an emerging technology that are under continued development. As such, they offer appealing opportunities for social interaction, educational applications, commercial activities (including training, public-relations, and marketing, in addition to e-commerce), as well as research and development. As one example, the following case study illustrates the utility of a 3D Digital Ecosystem in the research commercialisation of an Information Systems application.

\section{3D DIGITAL ECOSYSTEMS IN INFORMATION SYSTEMS RESEARCH COMMERCIALISATION}

3D Digital Ecosystems offer rich possibilities for research, development, and research commercialisation. While this is true for a variety of industries, it is highly pertinent for software development. This section describes a university Information Systems research, development, and commercialisation project that incorporates Second Life.

As an outgrowth of a collaborative research program regarding Automated Essay Grading (AEG) [22], an AEG program dubbed MarkIT (www.essaygrading.com) is being commercialised by Curtin University of Technology in a joint venture called Blue Wren Software. MarkIT uses a Natural Language Processing algorithm (the Normalised Word Vector algorithm) in conjunction with a thesaurus to analyse the semantic content of essays and to assign a mark in relation to a model answer [23].

While some forms of automated assessment are developing popularity (e.g., Turnitin; http://turnitin.com), these focus on matching text-strings for plagiarism-checking. In contrast, there are barriers to market-entry in Australia regarding semantic-based AEG. Consequently, the Automated Assessment Laboratory was formed at Curtin Business School to promote research and practice into the pedagogical benefits of AEG. Preliminary discussion with stakeholders suggests that barriers to use of AEG in Australia may be due to factors such as lack of information and trust regarding the AEG process and fears regarding educators' loss of jobs. In contrast, research suggests that the AEG process is as accurate as human markers [22], can promote significant financial savings, can improve feedback to students, and can free academics' resources to teach rather than mark.

Consequently, the Online Automated Assessment Laboratory was established in Second Life on the 'Australis 4 Learning' educational island. Its function is to promote the use of and benefit from AEG in educational institutions by enabling knowledge transfer and proof of concept in a 3D Digital Ecosystem. In Second Life, stakeholders can visit the interactive Online Automated Assessment Laboratory in order to learn more about the process (see Fig. 1). Additionally, a multimedia educational simulation depicts the AEG process and its pedagogical utility in tertiary contexts [24].

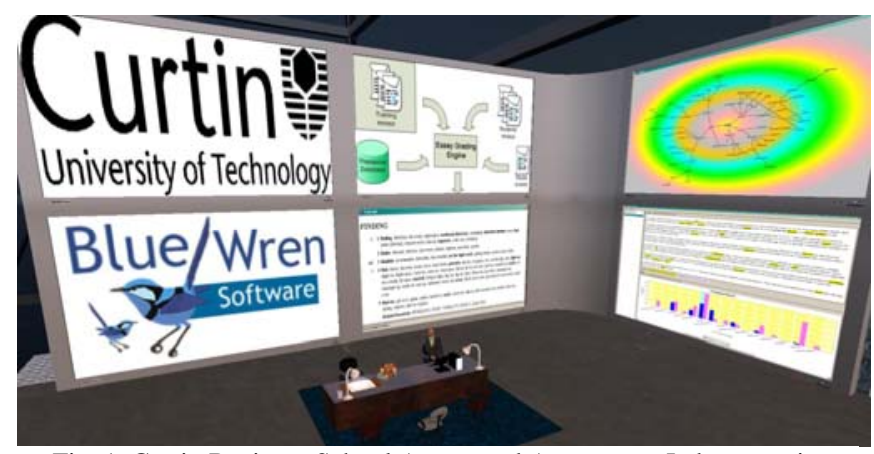

Fig. 1 Curtin Business School Automated Assessment Laboratory in Second Life

In addition to simulating the AEG process as a demonstration, the Online Automated Assessment Laboratory facility can complete the AEG process fully online. Students can submit electronic copies of their essays into an assignment submission box in Second Life (Fig 2.).

This facility both logs and authenticates userinteractions in order to regulate the accuracy and timeliness of students' submissions. For example students are only permitted to submit assignments for courses in which they are enrolled, and late assignments are automatically logged. The assignment submission box interfaces with a database external to Second Life, which in turn interacts with the AEG program. Students' individual results can be processed with minimal delay (i.e., within seconds to minutes) and interactive graphical feedback is provided to facilitate interactive formative assessment in addition to the grade as summative assessment [25] (Fig. 3). Aggregate group statistics are also provided after the due date for submission. Once students have had opportunity to peruse the AEG feedback, they are able to contact the lecturer if they have queries or desire assistance with course material.

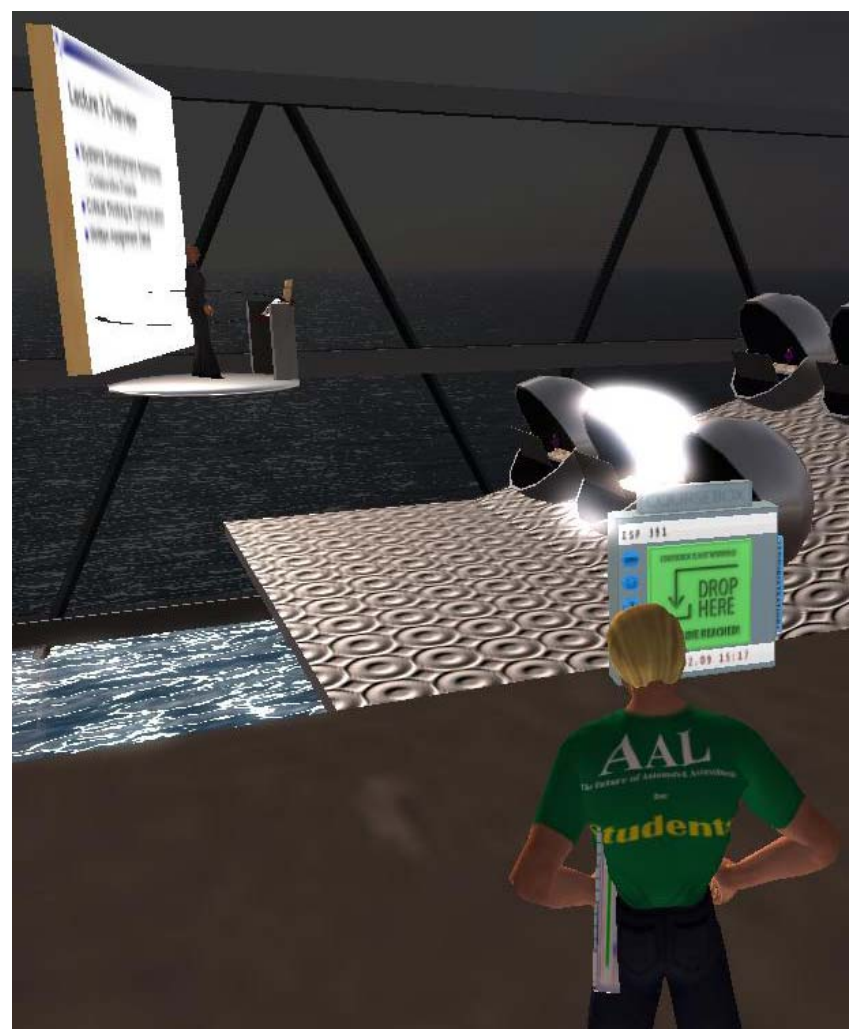

Fig. 2 Automated Assessment Laboratory Assignment Submission Box in Second Life 


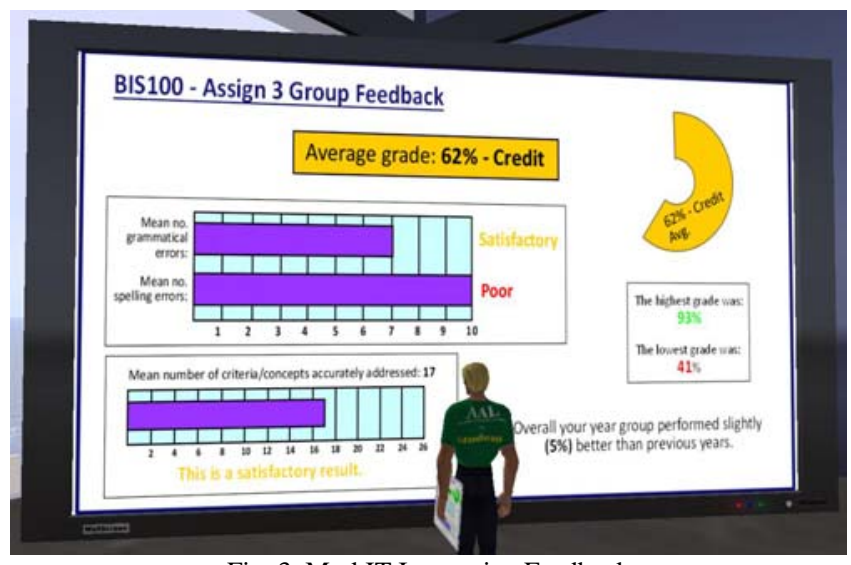

Fig. 3 MarkIT Interactive Feedback

In summary, the case study above illustrates the utility of using a 3D Digital Ecosystem as one means of augmenting a research commercialisation project by innovatively facilitating knowledge transfer and 'in-world' service provision of an emerging technology - Automated Essay Grading. Subsequent to this contextual introduction is a synopsis of the benefits and limitations of research and commercialisation that is enabled by 3D Digital Ecosystems.

\section{SYNOPSIS OF BENEFITS AND LIMITATIONS}

For organisations engaged in ICT research, development, and commercialisation (e.g., universities as well as commercial entities) there are certain benefits and limitations to the use of 3D Digital Ecosystems such as Second Life.

\section{A. Benefits of 3D Digital Ecosystems}

Benefits of using 3D Virtual Worlds in research, development, and commercialisation include: economic benefits, ICT-system advantages, enhanced collaboration, research productivity, and staying at the forefront of innovation.

There are a number of financial benefits to using Second Life in systems development and commercialisation projects. First, there are low start-up costs: Second Life facilitates zero-expense entry via a freeware client and free avatar accounts, and; purchasing land on which to conduct projects is relatively inexpensive [26]. Second, the virtual environment can provide improved planning/simulation before enacting a real-to-life physical system/structure: the flexible simulation capacities enable the prototyping or iterative modelling of physical structures, ICT systems, and business models that would be prohibitively expensive to experiment with in the real world. Third, the Second Life in-world currency is exceedingly inexpensive (approx USD\$1 to L\$260; [27]), and most in-world purchases are small (e.g., L\$10).

As an ICT-system, Second Life presents a number of advantages. First, because it is Internet-based and allows free access for all users, this overcomes licensing issues restricting the number of team-members who can work on the project simultaneously. Second, the interface is userfriendly; it requires minimal experience (e.g., approx. 10-20 hours) for proficiency in navigation and building functions. Third, the 'game-like' 3D interface and avatar-interaction is more interesting and intrinsically motivating than a text- editor programming application. Fourth, the system is highly flexible: the availability of the Linden Scripting Language has resulted in a constant stream of user-generated innovations (e.g., streaming video into the 3D Digital Environment).

Collaboration is enhanced within 3D Digital Ecosystems such as Second Life because: as a networked application all users can simultaneously see system developments as they are implemented; it also allows immediate application of one's development in context, which expedites iterative system testing and refinement; Second Life facilitates communication between team-members via text (synchronous and asynchronous), voice (local and remote), and body language (emotive facial expressions and body movements). Indeed it is remarkable that team-members can work simultaneously and communicate within the programming environment. These elements: promote a sense of immersion and presence and enhance communication, and; in the context of project development teams can foster team-spirit and aid effective collaboration.

Research productivity is also benefited. In the authors' own experience and through observing research students, there is a culture of enthused engagement and innovation in the community of users that literally generate the content of the Second Life ecosystem. This creativity spills out of Second Life into Web 2.0 applications such as wikis and blogs which provide comprehensive user-support. By engaging in the development of an emerging technology such as this, work becomes play, and innovative productivity is easily transferred into research projects and publications. Indeed, project students working in Second Life have been invited as guest speakers at conferences and published papers. When the cultures of the Second Life user-community and the academic community combine in encouraging research /development and presentations/publications, then we see that this promotes researchers' engagement and success in a research track. Importantly, in Second Life users own the intellectual property rights to their creations [28]. In short, research productivity is enhanced by the engaging 3D Digital Environment and by immersion in a rich culture of ICT innovation among the Second Life user-community. So while 3D Digital Ecosystems such as Second Life present a variety of benefits, they also present certain limitations and challenges.

\section{B. Limitations and challenging factors}

It is perhaps because 3D Virtual Worlds are an emerging technology that their current limitations relate to both technical and social factors. Technical limitations currently relate to: internet bandwidth requirements and the need for powerful computers [29]; the relatively large internet usage requirements in organisational contexts where usage is metered and charged for. However, in any organisation that is serious about supporting its $\mathrm{R} \& \mathrm{D}$, these considerations are no limitation whatsoever.

A social factor that limits the benefit gained by research organisations (esp. universities) from 3D Digital Ecosystems is that this emerging technology is rapidly evolving, both technologically and socially $[16,30]$. For instance, a challenge that universities face is for academics to stay at the forefront of innovation in industry and society general- 
ly, and for university policy makers to not only remove institutional barriers but to actively promote innovation by changing the structure of institutional ICT serviceprovision. As such, it is pertinent to consider the possibilities that 3D Digital Ecosystems present for future research, development, and commercialisation in Information Systems.

\section{Possibilities for Future RESEARCH, DEVELOPMENT, AND COMMERCIALISATION}

The continued growth of high connectivity computing in society and business will likely force ICT research-oriented institutions to keep pace with socio-technological advances lest they become increasingly irrelevant to community life and industry [5]. This is particularly relevant to universities, who need to: stay at the forefront of research to gain funding from the government and competitive grants, and; attract students in order to viably function as a commercial entity. If staying at the forefront of innovative ICT development is one's intention, then it is certainly important to study current trends and to prepare for a range of possibilities (e.g., by upgrading hardware and 'up-skilling' staff in industry-relevant skills). Consequently, the final section of this paper regards emerging, possible, and desirable advances in ICT and Information Systems research and development across the next 5 to 10 years.

Current innovations in Second Life that are only beginning to be implemented include: pedagogical applications such as smart lecture theatres and classrooms that function as educational support systems by linking the real and virtual worlds to facilitate blended learning and distance learning; human space computing applications such as intuitive navigation using 3D mice and/or the Nintendo Wii Balance Board and controller to enhance the immersive experience, using Nintendo Wii controllers to create interactive whiteboards ('smart boards'), and hosting Second Life on 3G mobile devices.

Potential future technological developments will further extend human space computing applications to include the use of body-motion sensors, hand-motion gloves, and virtual reality goggles to navigate in 3D Digital Ecosystems for a more immersive experience. Such developments could promote more interaction between real and virtual environments and benefit interaction in: classrooms in pedagogical contexts; systems development teams in research and development contexts, and; employees and trainers in vocational training applications. For example, the use of wireless/mobile technology could allow educators and students to be mobile around the classroom while connected to Second Life by using various output devices (e.g., projector/lecture screens, 'virtual-reality-goggles') and various innovative (3D) input devices. The advent of mobile phones that sense hand-held motion (via accelerometers) and geographic location (via global positioning satellites) presents intriguing possibilities for facilitating in-world navigation through movements in the real-world, especially when combined with virtual reality headsets with stereoscopic (3D) vision. Such developments are the future of human space computing. In this vein, a rich field for development is education and training through simulations and games.

Educational games are a rich and largely untapped market. Their applications are as relevant to businesses for vocational training as they are to educational institutions. The profit inherent in the gaming industry rivals that of the movie or music industries [31]. Game users frequently report that computer games are highly motivating [32], which is an element often missing from modern educational contexts. Games also offer unique opportunities to learn skills. The development of educational simulations and games can be used to provide structured reinforcement to develop useful skills (e.g., critical/creative thinking). Second Life is a useful candidate for such developments due to its accessibility and flexibility.

These game-like applications in Second Life could perform a number of functions, including: (a) teaching content (as per standard educational settings); (b) developing skills (i.e., through constructivist learning principals, as in project units etc.), and; (c) assessing performance for academic assessment (formatively or summatively).

Various educational outcomes of such game-like applications could include: (a) teaching in-world Second Life skills such as navigation, programming gestures, social interaction (acculturation), building objects ('primitives' and 'sculpties'), and the LSL programming language; (b) teaching creative and analytical thinking; (c) developing cognitive executive functions (i.e., attentional control, sustained attention, attention switching); (d) teaching goal setting and emotional intelligence skills, such as conflict resolution skills and delayed gratification (emotional intelligence skills predict real-world success [33]); (e) teaching prosocial online cultural norms and ethical values (using avatars to personalise the usually depersonalised online contexts); (f) teaching and developing project management skills; (g) developing programming skills (e.g., Visual Basic, Java \& $\mathrm{C}++$ ), and; (h) teaching and developing entrepreneurial skills through establishing actual businesses in Second Life interfacing the real-world via the World Wide Web. In short, the development of educational games offers rich pedagogical and financial benefits for educational institutions and commercial organisations alike. Indeed, educational gaming could develop into a profitable industry.

In summary, there is great potential inherent in the use of 3D Digital Ecosystems for information systems technology research, development, and commercialisation. Such developments will keep pace with the digital-native culture of younger generations and have the potential to innovatively revolutionise our social systems relating to governance, education, commerce, and social interaction. Digital Ecosystems, 3D Virtual Worlds in particular, are set to lead the charge in our modern culture of accelerating innovation. 


\section{REFERENCES}

1 J.V.C. Nye, "Standards of living and modern economic growth," 2008, Retrieved February 14, 2009, from http://www.econlib.org/ library/Enc/StandardsofLivingandModernEconomicGrowth.html

2 G.E. Moore, "Cramming more components onto integrated circuits," Electronics, vol. 38, no. 8, 1965, pp. 114-117.

3 E. Mollick, "Establishing Moore's Law," IEEE Annals of the History of Computing, vol. 28, no. 3, 2006, pp. 62-75.

4 P. Drucker, The age of discontinuity: Guidelines to our changing society, $1^{\text {st }}$ ed., New York: 1969.

5 M. Pesce, "Inflection points," 2008, Retrieved January 19, 2009, from http://blog.futurestreetconsulting.com/

6 Wikipedia, "Internet censorship in the People's Republic of China," 2009, Retrieved February 14, 2009, from http://en.wikipedia.org/ wiki/Internet_censorship_in_the_People\%27s_Republic_of_China

7 D.E. Bambauer, "Filtering in Oz: Australia's forey into Internet censorship," 2008, Retrieved February 14, 2009, from http://ssrn.com/abstract=1319466

8 Free Software Foundation, "GNU operating system," 2009, Retrieved February 14, 2009, from http://www.gnu.org/

9 D. Boyd, "Why youth (heart) social network sites: The role of networked publics in teenage social life," in MacArthur Foundation Series on Digital Learning - Youth, Identity, and Digital Media Volume, D. Buckingham (Ed.), Cambrige, MA: 2007, pp.

10 National Research Priorities Standing Committee, National research priorities report to Government, Commonwealth of Australia, Canberra, ACT, Australia: 2007, Retrieved February 14, 2009, from http://www.dest.gov.au/sectors/research sector/publications resources /profiles/documents/NRP_report_to government_pdf.htm

11 J. Howard, The emerging business of knowledge transfer: Creating value from intellectual products and services, Commonwealth of Australia, Canberra, ACT, Australia: 2005, Retrieved February 14, 2009, from http://www.dest.gov.au/sectors/research_sector/policies_ issues_reviews/key_issues/commercialisation/documents/knowledge_ transfer_pdf.htm

12 HowardPartners, Best practice processes for university research commercialisation, Commonwealth of Australia, Canberra, ACT, Australia: 2002, Retrieved February 14, 2009, from http://www.dest.gov.au/sectors/research_sector/publications_resources /indexes/documents/bestpractice_x2_pdf.htm

13 PhillipsKPA, Knowledge transfer and Australian universities and publically funded research agencies: A report to the Department of Education, Science and Training, Commonwealth of Australia, Barton, ACT, Australia: 2006, Retrieved February 14, 2009, from http://www.dest.gov.au/sectors/research sector/policies issues revie ws/key_issues/commercialisation/knowledge_transfer.htm\#Full_Repo $\mathrm{rt}$

14 Wikipedia, "History of Google," 2009, Retrieved February 14, 2009, from http://en.wikipedia.org/wiki/History_of_Google
15 J. Fenn, "Survey shows adoption and value of emerging technologies," in Gartner Research, Gartner Inc., Stamford, CT: 2006, ID Number: G00138453.

16 A. Sarner, "The business impact of social computing on marketing and 'Generation Virtual'," in Gartner Research, Gartner Inc., Stamford, CT: 2008, ID Number: G00161081.

17 D.M. Smith, T. Austin, G. Phifer, C. Da Rold, M.W. Cain and Y.V. Natis, "Predicts 2009: Cloud computing beckons," in Gartner Research, Gartner Inc., Stamford, CT: 2008, ID Number: G00163522.

18 S. Prentice, "The five laws of virtual worlds," in Gartner Research, Gartner Inc., Stamford, CT: 2007, ID Number: G00148019.

19 G. Constable, "Virtual Worlds: A short introduction to Second Life," 2008, Retrieved January 16, 2009, from http://au.youtube.com/watch?v=5ME2FwjiPf8

20 R.V. Kail and J.C. Cavanaugh, Human development: A lifespan view, 2nd ed., Belmont, CA: 2000.

21 C. Abrams, "Three ways that Second Life can add real benefits for Web business, despite the negative hype," in Gartner Research, Gartner Inc., Stamford, CT: 2007, ID Number: G00153458.

22 R. Williams and H. Dreher, "Automatically grading essays with Markit@," Journal of Issues in Informing Science and Information Technology, vol. 1, no. 2004, pp. 693-700.

23 R. Williams, "The power of Normalised Word Vectors for automatically grading essays," The Journal of Issues in Informing Science and Information Technology, vol. 3, no. 2006, pp. 721-730.

24 N. Dreher, C. Dreher, H. Dreher and T. Reiners, "Australis 4 Learning machinima," 2008, Retrieved February 1, 2008, from http://eaglesemantics.com/node/257

25 H. Dreher, "Interactive on-line formative evaluation of student assignments," Issues in Informing Science and Information Technology, vol. 3, no. 2006, pp. 189-197.

26 LindenResearch, "Private region pricing," 2009, Retrieved February 1, 2009, from http://secondlife.com/land/privatepricing.php

27 LindenResearch, "L\$ marketplace," 2008, Retrieved 14 February 2009, from http://wiki.secondlife.com/wiki/L\$_Marketplace

28 LindenResearch, "IP Rights," 2009, Retrieved January 17, 2009, from http://secondlife.com/whatis/ip rights.php

29 LindenResearch, "System requirements," 2009, Retrieved February 1, 2009, from http://secondlife.com/support/sysreqs.php

30 S. Prentice and A. Sarner, "Defining Generation V: The virtual generation," in Gartner Research, Gartner Inc., Stamford, CT: 2008, ID Number: G00154114.

31 Seeking Alpha, "The video game industry: An \$18 billion entertainment juggernaut," 2008, Retrieved February 14, 2009, from http://seekingalpha.com/article/89124-the-video-game-industry-an-18billion-entertainment-juggernaut

32 P. Vorderer, T. Hartmann and C. Klimmt, "Explaining the enjoyment of playing video games: The role of competition," in Proceedings of the Second ACM International Conference on Entertainment Computing 2003, pp. 1-9.

33 D. Goleman, R. Boyatzis and A. McKee, Primal leadership: Realizing the power of emotional intelligence, $1^{\text {st }}$ ed., Boston: 2002. 